\title{
PROGRESS IN THE OPERATION OF A HIGH INTENSITY EBIS AT BNL*
}

\author{
J.G. Alessi ${ }^{\dagger}$, E. Beebe, O. Gould, A. Kponou, A. Pikin, K. Prelec, J. Ritter, \\ BNL, Upton, NY 11973, USA
}

\begin{abstract}
Excellent progress has been made in the operation of the BNL Electron Beam Ion Source (EBIS), which is a prototype for an EBIS that could meet requirements for a RHIC preinjector. We have achieved very stable operation of the electron beam at $10 \mathrm{~A}$ through the EBIS trap. At 7 A electron beam current, $2.8 \times 10^{11}$ charges have been extracted in short pulses from ionization of residual gas, exceeding our goal for yield for the corresponding trap capacity. With Au injection, we have achieved $25 \%$ of the RHIC intensity requirement with only 4 A electron current, and half the final trap length.
\end{abstract}

\section{INTRODUCTION}

The present preinjector for heavy ions for AGS/RHIC uses the Tandem Van de Graaff. An alternative to this can be an Electron Beam Ion Source (EBIS), followed by a Radiofrequency Quadrupole accelerator and a short Linac. This new preinjector offers improvements in both performance and operational simplicity. In this case, one would produce, directly from the ion source, the charge state desired for Booster injection. This eliminates the particle loss from any subsequent stripping efficiencies, and makes the initial preacceleration more efficient. In addition, Booster injection will be more efficient if one can inject over fewer turns than presently used, so it is also desirable for the source to produce shorter pulses of higher currents. Some of the source requirements are:

- Intensity: $3.4 \times 10^{9} \mathrm{Au}^{32+}$ ions per pulse

- Pulse width: variable, $10-40 \mu$ s, to allow 1-4 turn injection into the Booster

- Repetition rate: $5-10 \mathrm{~Hz}$

- $\mathrm{q} / \mathrm{m}: 0.16$ or greater. Equals that presently used for $\mathrm{Au}$ from the Tandem. For lighter ions, higher $\mathrm{q} / \mathrm{m}$ is required $\left(\mathrm{Si}^{14+}, \mathrm{Fe}^{21+}\right)$ to achieve the desired Booster output energy.

We have chosen to develop an EBIS to meet the above requirements. The charge state requirements are modest for an EBIS. An EBIS delivers pulses having a constant total positive charge, and one has control over the ion pulse width by controlling the trap voltages. Ions can be extracted in short pulses of high current, which is desirable for synchrotron injection. The narrow charge state distribution from an EBIS has an advantage vs. other types of high charge state heavy ion sources when considering the beam transport line. For the same current in the desired charge state, one has to deal with much higher total extracted currents in sources having broad charge state distributions, which can lead to space charge problems in transport. For example, $3.4 \times 10^{9} \mathrm{Au}^{32+}$ ions in a $10 \mu \mathrm{s}$ pulse corresponds to a $\mathrm{Au}^{32+}$ current of $1.7 \mathrm{~mA}$. The total extracted current from the EBIS will be $8.5 \mathrm{~mA}$, assuming a reasonable value of $20 \%$ in the desired charge state. For sources such as the ECR or Laser Ion Source, this total current could be at least an order of magnitude higher for the same yield of $\mathrm{Au}^{32+}$. Finally, an EBIS can easily produce ions of any species, and can rapidly switch between species (even pulse-to-pulse).

A conservative approach has been chosen for the RHIC EBIS concept. The EBIS would operate in a "traditional" mode, have a $1.5 \mathrm{~m}$ long trap, a warm bore, an unshielded superconducting solenoid, and a $10 \mathrm{~A}, 20 \mathrm{keV}$ electron beam with current density $<600 \mathrm{~A} / \mathrm{cm}^{2}$. The goal ion yield of $3.4 \times 10^{9}$ ions of $\mathrm{Au}^{32+}$ will be reached with the above parameters if the neutralization of the electron beam by the ion species of interest is $50 \%$, a value exceeded in the BNL EBIS, as well as in other EBISs. It is also assumed that $20 \%$ of that charge is in the desired charge state, again a condition frequently achieved in EBISs. The recent progress at BNL in demonstrating the required performance is presented below.

\section{DESCRIPTION OF EBTS}

The EBIS Test Stand (EBTS) is a full-power, half length prototype of the RHIC EBIS. It has the following main components:

SC solenoid: A $1 \mathrm{~m}$ long, unshielded, warm bore magnet with an inner diameter of $154 \mathrm{~mm}$. The solenoid, manufactured by Oxford Instruments, Inc., has a maximum operating field of $5 \mathrm{~T}$.

Drift structure: This consists of 12 insulated stainless steel tubes (32 mm ID), sitting at room temperature. The four central tubes form an ion trap of length $71 \mathrm{~cm}$.

Electron Collector: This is water cooled, and designed for a maximum average electron beam power dissipation of 50 $\mathrm{kW}$. The collector is partially surrounded by an iron magnetic shield, and a coil in front of the collector is used to adjust the field at the entrance. Operating the electron beam in pulsed mode $(100 \mathrm{~ms})$, the collector has operated at instantaneous powers in excess of $120 \mathrm{~kW}$.

Electron gun: The design of the electron gun was of crucial importance not only because of the requirement for such a high current, but also because of the need for a flexible control of the electron beam parameters. The gun has an 8.3 $\mathrm{mm}$ diameter, single crystal $\mathrm{LaB}_{6}$ cathode, and is a coaxial diode with magnetic insulation, positioned in the field of a separate solenoid. The gun was designed and fabricated at the Budker Institute of Nuclear Physics, Novosibirsk [1]. 


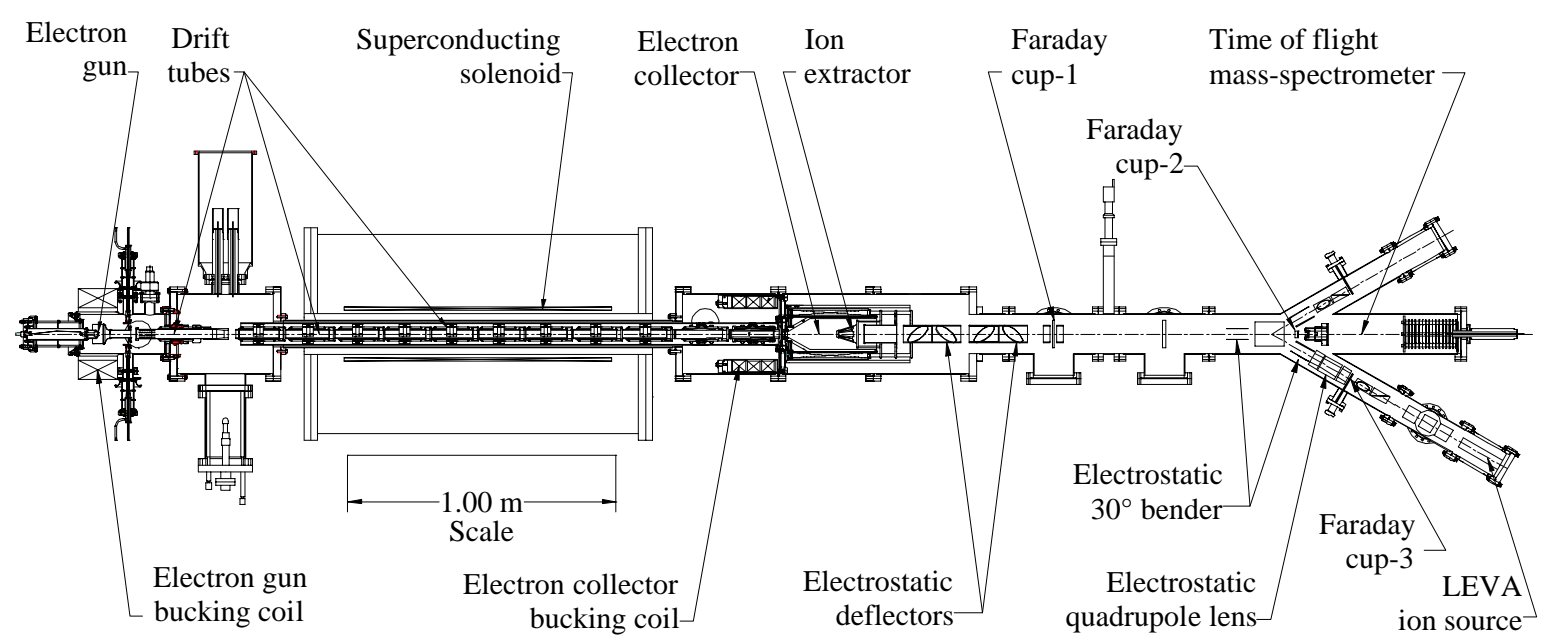

Figure 1: Schematic of the EBIS test stand and ion injection.

There are separate bucking coils for the electron gun and electron collector, which decouple the electron beam launching, compression and collection from the main solenoid. Five sets of external transverse magnet coils allow versatile adjustment of the electron beam transmission in different regions, and their use significantly reduces electron beam losses on elements of the drift structure.

EBTS operates in the pulsed mode for both the electron gun and the drift tube high voltages, which extends the range of our $50 \mathrm{~kW}$ electron collector, and allows us to freely explore source parameters while still maintaining ultrahigh vacuum. A detailed description of the EBTS design and earlier results is presented in [2].

\section{SUMMARY OF PREVIOUS EBTS RESULTS}

The performance of the novel electron gun has been excellent. It has provided very stable operation over a wide range of gun operating parameters. With this gun we have reached our design goal, and propagated a $10 \mathrm{~A}$ electron beam through the EBIS solenoid to the collector, with very low beam loss $(<0.5 \%)$, in $10 \mathrm{~ms}$ pulses. We have also operated with an $8.6 \mathrm{~A}, 100 \mathrm{~ms}$ electron beam (current-limited at the time by the anode power supply)

Our design goal of extraction of a total ion charge corresponding to $50 \%$ of the electron beam space charge has consistently been met or exceeded. Trapping and ionization of background gas ions was the most straightforward first test of operation, with the ion yield given in Table 1. Gas injection was then tried, although EBTS was not designed for this since it does not have a convenient way to separate the gas injection region from the main trap region. Injection becomes constant neutral gas injection, which leads to a broader charge state distribution. (We prefer to use the alternative external ion injection scheme). The ion yield for Xe gas injection is also given in Table 1. The RHIC EBIS requires a yield of $5 \times 10^{11}$ positive charges/pulse, at $10 \mathrm{~A}$ and at twice the trap length of EBTS, so our results to date have exceeded the EBTS design goal.
TABLE 1. Ion yields from EBTS

\begin{tabular}{|l|c|c|c|}
\hline \multicolumn{1}{|c|}{ Ion } & $\mathbf{I}_{\mathrm{e}}$ & charges/pulse & Neutralization \\
\hline Bkgnd gas & $7 \mathrm{~A}$ & $2.8 \times 10^{11}$ & $85 \%$ \\
\hline Xenon & $7 \mathrm{~A}$ & $1.9 \times 10^{11}$ & $55 \%$ \\
\hline $\mathrm{Au}$ & $4 \mathrm{~A}$ & $1.4 \times 10^{11}$ & $62 \%$ \\
\hline
\end{tabular}

We can not yet measure ion yields at the full electron beam current. Practical limitations to the voltages that can be applied to the trapping electrodes have prevented trap formation when one has a deeper potential well from the higher electron beam currents. In the future, planned design modifications and power supply upgrades will allow extraction of ions from a 10 A electron beam.

For 1-4 turn injection into the Booster, the extracted ion pulse should be $10-40 \mu \mathrm{s}$ long. Using a 6 A electron beam, a $10 \mu \mathrm{s}$ FWHM ion pulse was extracted from EBTS, having a peak ion current of $3.3 \mathrm{~mA}$. The yield was $1.9 \times 10^{11}$ charges, corresponding to $57 \%$ neutralization. This result was achieved by raising the voltage of the trap region above the level of the barrier electrode, with an additional voltage tilt in the trap produced via a resistor/capacitor network. While this result was with ions produced from background gas, this fast extraction can be similarly achieved for any ions. Once demonstrated, however, the fast power supplies used were reconfigured for use for ion injection experiments, to be described in the next section.

Finally, charge state distributions were measured using time-of-flight (TOF) for Ar, Xe, and Cs. The distributions are consistent with the expected values based on the electron current density and ion confinement time.

\section{RECENT RESULTS WITH EXTERNAL INJECTION OF AU IONS}

The ion optical system, shown in Fig. 1 is used both for injection of singly charged ions into the EBIS trap from an auxiliary ion source, and for diagnostics of the ion beam extracted from EBTS. The transport line includes $30^{\circ}$ pulsed electrostatic deflectors, which allow use of multiple auxiliary sources and provides for time-of-flight (TOF) analysis of the extracted EBTS beam in the straight section. The injected ion beam was monitored with 
removable Faraday cup FC3 at the exit of Au ion source, and FC1, close to the exit from EBTS. The extracted ion beam from the EBTS was measured on $\mathrm{FC} 2,160 \mathrm{~cm}$ from the exit of EBTS.

$\mathrm{Au}^{1+}$ ions were produced in a LEVA source [3] having 7 apertures, each of $\sim 1.5 \mathrm{~mm}$ diameter. These ions were extracted in $\sim 500 \mu$ s pulses at $10 \mathrm{kV}$. "Fast" injection was used. With an initial flat potential distribution on trap drift tubes, ions of $\mathrm{Au}^{1+}$ make a round trip traversal of the trap region, reflecting from the gun barrier. A flat potential distribution is imposed in the trap region and the potential can be adjusted to retard the injected $\mathrm{Au}^{1+}$ beam to $<100$ $\mathrm{eV}$ in the EBTS trap region, thereby increasing the linear charge density of the injected $\mathrm{Au}^{1+}$ beam. With the $\mathrm{Au}^{1+}$ ion beam present, the potential on the trap drift tubes is then lowered, resulting in axial trapping of traveling ions, which find themselves confined between two axial barriers. In this method of injection, ions do not have to be further ionized to be trapped; therefore the efficiency of trapping can be high and the injection times are rather short. The LEVA produced more than enough ions. One would typically fill the trap to $10-20 \%$ of capacity with $\mathrm{Au}^{1+}$, and then start the stepwise ionization.

Figure 2 shows $\mathrm{Au}$ ion yields measured on a $2.3 \mathrm{~cm}$ diameter Faraday cup located $1.6 \mathrm{~m}$ from the extractor. The yield as a function of electron current exceeds the goal of 50\% neutralization. The measured yield of $1.4 \mathrm{x}$ $10^{11}$ charges per pulse at $4 \mathrm{~A}$ electron beam current corresponds to a $62 \%$ neutralization. The $\mathrm{Au}$ ion results were taken at currents up to only $4 \mathrm{~A}$ because a vacuum leak prevented the water cooling of the collector. This leak has now been repaired, and measurements will soon continue at higher electron currents.

Figure 3 shows the ion yield vs. confinement time at various electron currents. Figure 4 shows a $\mathrm{Au}$ TOF spectrum measured at $2.8 \mathrm{~A}$ and $32 \mathrm{~ms}$ ion confinement time with a most abundant charge state of $\mathrm{Au}^{27+}$. This shows the desired narrow charge state distribution, and the charge state reached is consistent with that required when scaling up to $10 \mathrm{~A}$ and longer confinement time.

\section{CONCLUSIONS}

The output of EBTS is now less than a factor of 2 below the RHIC requirement for charge, with half the final trap length - demonstrating proper operation of an EBIS at high currents. At only $4 \mathrm{~A}$ electron current, the $\mathrm{Au}$ ion intensity was $25 \%$ of the final RHIC requirement. To date, all results of the EBTS have agreed with EBIS scaling laws, and continue to confirm the parameters for a RHIC EBIS that were presented about 10 years ago.

The progress made at BNL on the EBIS development has increased our confidence that such a source injecting into a Linac-based preinjector can reach the pulsed $\mathrm{Au}$ beam intensity required for RHIC, and offers significant advantages in meeting long-term requirements for performance and reliability for the RHIC program.

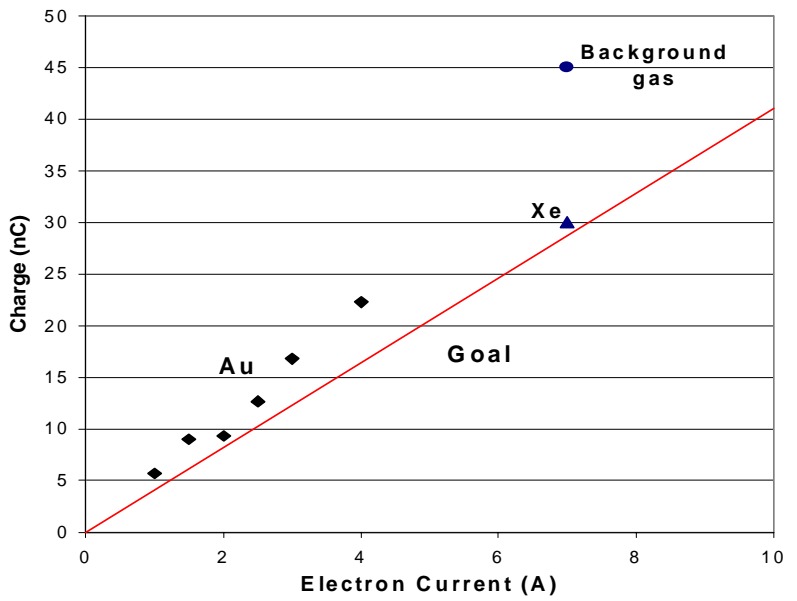

Figure 2: Ion yield vs. electron current.

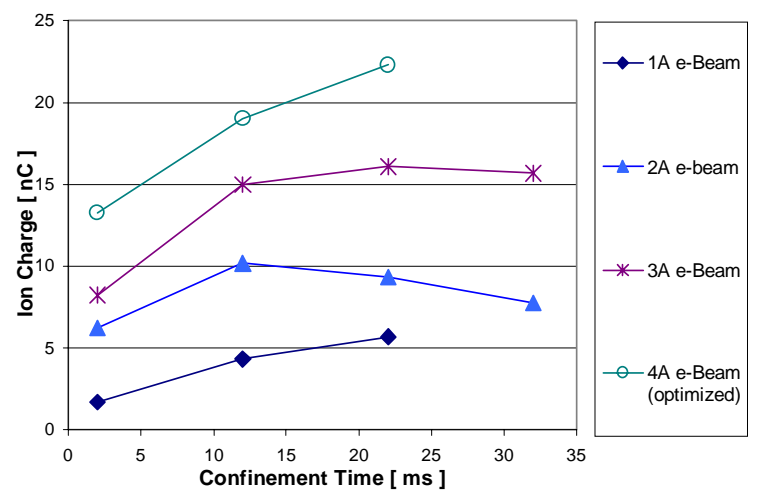

Figure 3: Au ion yield vs. confinement time, at various electron currents.

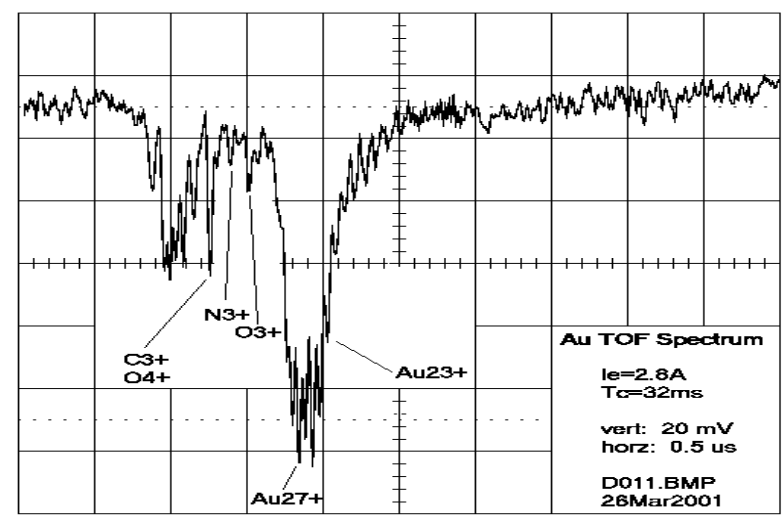

Figure 4: TOF spectrum for gold external ion injection into the EBTS ( $\mathrm{Ie}=2.8 \mathrm{~A}$, confinement time $=32 \mathrm{~ms}$ ).

\section{ACKNOWLEDGEMENTS}

We would like to thank D. Boeje, D. Cattaneo, D. Graham, R. Lockey, D. McCafferty, R. Schoepfer, and W. Shaffer, for their excellent technical support.

\section{REFERENCES}

[1] A. Kponou, et.al., Rev. Sci. Instrum. 69, (1998) 1120. [2] A. Pikin, et.al., "EBTS: Design and experimental study", Proc. $8^{\text {th }}$ Int. Symp. on Electron Beam Ion Sources and Traps (Nov., 2000), AIP Conf. Proc., to be published. [3] I.G. Brown, et.al., Rev. Sci. Instrum. 65 (1994) 1260. 